American Journal of Applied Sciences 4 (1): 53-55, 2007

ISSN 1546-9239

(C) 2007 Science Publications

\title{
On Thermal Explosion of Sensitized Reaction with Variable Heat Loss
}

\author{
K. S. Adegbie and F. I. Alao \\ Department of Mathematical Sciences, Federal University of Technology, Akure, Nigeria
}

\begin{abstract}
This study discusses the influence of heat loss on the critical ignition temperature and Frank-Kamenetskii parameter of a non-linear ordinary differential equation arising in thermal sensitized reaction. The reaction obeys the Arrhenius expression with temperature dependent preexponential factor, taking heat exchange between the reacting material and its surrounding into account. The consequences of heat loss are explored within the framework of one dimensional, steady state model. The numerical estimations based on shooting method techniques show the effect of heat loss parameter on the critical values of ignition temperature and Frank-Kamenetskii parameter.
\end{abstract}

Key words: Frank-Kamenetskii parameter, thermal sensitized reaction, critical ignition temperature

\section{INTRODUCTION}

The problems of thermal explosion have been widely discussed in literatures Semenov ${ }^{[1]}$ was perhaps the first to develop the basic theory of the phenomenon of thermal explosion, Dainton ${ }^{[2]}$, Frank-Kamenetskii ${ }^{[3]}$. Since that time more and more complicated models have been suggested (Zeldovich et al. $.^{[4]}, \mathrm{Kuo}^{[5]}$, Boddington et al. ${ }^{[6]}$ and related references therein). The problems of exothermic chemically reactive material in a slab obeying an Arrhenius law and accounting for temperature dependence of pre-exponential factor $\mathrm{A}(\mathrm{T})$ are based on the assumptions that the reacting material is motionless, that the heat losses are purely determined by the thermal conductivity of the reacting material and that the chemical reaction proceeds non-uniformly over the vessel volume (Ayeni $^{[7]}$, Okoya ${ }^{[9,10]}$, Ajadi $^{[11]}$ and related references therein).

The analyses of these problems have been performed based on close-form, approximation and phase-plane methods and numerical techniques using computational fluid dynamics (CFD) packages. In another development, Ayeni et al. ${ }^{[8]}$ examined the effect of heat loss on ignition times in the theory of branched chain explosions and showed how severe the heat loss could be to prevent ignition from occurring.

Recently, Okoya ${ }^{[10]}$ considered the steady-state solutions for the exothermic chemical reaction, taking the diffusion of the reactant in a slab into account. He presented a new analytical solution for the FrankKamenetskii parameter $\delta$ in the special case corresponding to the 'Sensitized' reaction. This analytical solution, as it is in terms of Bernoulli's numbers, is in accordance with the numerical integration for $\varepsilon(<<1)$ is very small and for $\varepsilon \rightarrow 0$ it boils down to the solution for the Frank-Kamenetskii ${ }^{[3]}$.
Also the transitional values of $\delta, \varepsilon$ and $\theta_{\mathrm{m}}$ were determined numerically. In the specific problems of thermal explosion discussed above, the establishment of the proper heat transfer conditions, which depend on the physical circumstances of the process, is of great importance in industries. The ignition temperature is the temperature to which a combustible material must be raised before it begins to burn. In fact, the greatest temperature for which a low temperature steady-state distribution is possible is known as the critical ignition temperature or critical storage temperature. At temperature higher than the critical ignition temperature, thermal ignition will occur.

The present work extends Okoya ${ }^{[10]}$ model to account for effect heat loss on critical ignition temperature and Frank-Kamenetskii parameter.

2. Mathematical formulation: We consider an exothermic thermal sensitized reaction in a closed vessel whose walls are maintained at a fixed temperature $T_{0}$ throughout the process. At the beginning the material temperature is also $T_{0}$. The rates of heat release in the vessel and heat transfer to the vessel walls are $q_{+}=Q A(T) \exp (-E / R T)$ and

$q_{-}=\alpha S / V\left(T-T_{0}\right)$ respectively. The conservation equation under the one-dimensional steady-state assumption in which heat exchange between the reacting material and the slab walls has the following form

$\lambda \frac{\mathrm{d}^{2} \mathrm{~T}}{\mathrm{~d} \overline{\mathrm{x}}^{2}}+\rho \mathrm{QA}\left(\frac{\mathrm{KT}}{\mathrm{vh} \rho}\right)^{\mathrm{r}}$
$\exp \left(\frac{-\mathrm{E}}{\mathrm{RT}}\right)-\alpha \frac{\mathrm{S}}{\mathrm{V}}\left(\mathrm{T}-\mathrm{T}_{0}\right)=0$

together with the boundary conditions

$\mathrm{T}=\mathrm{T}_{0} \quad$ on $\overline{\mathrm{x}}= \pm \mathrm{a}$

Corresponding Author: $\quad$ K. S. Adegbie, Department of Mathematical Sciences, Federal University of Technology, Akure, Nigeria 
where $\lambda$ is the thermal conductivity of the material, $\mathrm{Q}$ is the heat of reaction, A is the rate of constant, $v$ is the vibration frequency, $h$ is the Plank's constant, $\rho$ is the density, $r$ is a numerical exponent and $\mathrm{r} \in\{-2,0,0.5\}, E$ is the activation energy, $R$ is the universal gas constant, $T_{0}$ is the initial temperature, $\alpha$ is the convection coefficient, $S$ and $V$ are the surface area and volume of the slab respectively.

3. Method of solution: In order to reduce the number of parameters in equations (2.1) and (2.2), we introduce the following dimensionless variables:

$\theta=\frac{T-T_{0}}{\varepsilon T_{0}}, \varepsilon=\frac{R T_{0}}{E}, x=\frac{\bar{x}}{z}$.

Substituting equation (3.1) into equations (2.1) and (2.2) yields

$\frac{d^{2} \theta}{d x^{2}}+\delta(1+\varepsilon \theta)^{r} \exp \left(\frac{\theta}{1+\varepsilon \theta}\right)-\beta \theta=0$

and

$\theta=0$ on $x= \pm 1$

where $\delta=z^{2} \rho Q E A\left(k T_{0} / v h \rho\right)^{r} \exp \left(-E / R T_{0}\right) / \lambda R T_{0}^{2}$ is Frank-Kamenetskii parameter which measures the exothermicity of the reaction $(Q)$, the dimensions of the slab involved $\left(z^{2}\right)$ and the effect of the ambient (initial) temperature on the slab $\left(T_{0}\right), \beta=z^{2} \alpha S / \lambda$ is heat loss parameter and $\varepsilon$ is the activation energy parameter. The factors that influence the thermal ignition of the combustible materials depend on the parameters in equation (3.2), which are of great importance from the point of view of the safety techniques of handlings explosives and in thermal combustion industries.

It is worth mentioning that for $\beta=0$ and $r=0$, we get Frank-Kamenetskii's model for thermal explosion of exothermic reaction corresponding to "Arrhenius" form in the limit of large activation energy $(\varepsilon \rightarrow 0)^{[3]}$, we recover the classical simple closed-form solution of the form

$$
\theta=\theta_{m}+\ln \sec h\left\lfloor\left(\sqrt{\delta \exp \left(\theta_{m}\right) / 2}\right) x\right\rfloor
$$

where $\theta_{m}$ is the dimensionless temperature at the center of the slab. On employing the boundary condition (3.3), we have

$$
\delta=2 \exp \left(-\theta_{m}\right)\left\{\cosh ^{-1}\left(\exp \left(\theta_{m} / 2\right)\right)\right\}^{2}
$$

More generally, for $\beta=0$ and $r=-2$, a new analytical solution for Frank-Kamenetskii parameter $\delta$ corresponding to the "Sensitized" reaction for which $0<\varepsilon<<1$ was presented in form
Table 1: When $\beta=0$

\begin{tabular}{lll}
\hline$\varepsilon$ & $\delta_{\text {cr }}$ & $\theta_{\mathrm{m}, \mathrm{cr}}$ \\
\hline 0 & 0.87845 & 1.1868 \\
0.01 & 0.90520 & 1.1799 \\
0.05 & 1.01171 & 1.1538 \\
0.1 & 1.14378 & 1.1248 \\
\hline
\end{tabular}

Table 2: When $\beta=0.0525$

\begin{tabular}{lll}
\hline$\varepsilon$ & $\delta_{\text {cr }}$ & $\theta_{\text {m,cr }}$ \\
\hline 0 & 0.89716 & 1.1849 \\
0.01 & 0.92441 & 1.1778 \\
0.05 & 1.03282 & 1.1513 \\
0.1 & 1.16707 & 1.1218 \\
\hline
\end{tabular}

Table 3: When $\beta=1.1003$

\begin{tabular}{lll}
\hline$\varepsilon$ & $\delta_{\text {cr }}$ & $\theta_{\text {m.cr }}$ \\
\hline 0 & 1.27109 & 1.1450 \\
0.01 & 1.30671 & 1.1356 \\
0.05 & 1.44964 & 1.1012 \\
0.1 & 1.62225 & 1.0645 \\
\hline
\end{tabular}

Table 4: When $\beta=6.2214$

\begin{tabular}{lll}
\hline$\varepsilon$ & $\delta_{\text {cr }}$ & $\theta_{\text {m.cr }}$ \\
\hline 0 & 3.04020 & 0.9431 \\
0.01 & 3.10138 & 0.9291 \\
0.05 & 3.32962 & 0.8821 \\
0.1 & 3.58821 & 0.8366 \\
\hline
\end{tabular}

$\delta=2(1+\varepsilon \theta)^{4} \exp \left(\frac{-\theta_{\mathrm{m}}}{1+\varepsilon \theta_{\mathrm{m}}}\right)$

$\left(1-4 \varepsilon\left(1+\varepsilon \theta_{m}\right)\left\{\begin{array}{l}\ln \cosh p- \\ \sum_{n=1}^{\infty} \frac{(-1)^{n-1} 2^{2 n}\left(2^{2 n}-1\right) B_{n}}{(2 n+1) !} p^{2 n}\end{array}\right\}\right)^{2}+$ e.t.

for $|p| \leq \pi / 2$

Here $p=\cosh ^{-1}\left(\exp \left(\theta_{m} / 2[1+\varepsilon \theta]\right)\right)$

and $B_{n}$ are the Bernoulli's numbers and e.t. represent error terms. Previous study on thermal explosion involving perturbation and numerical analysis were presented in references stated in Okoya ${ }^{[10]}$.

It is worth noting that equations (3.2) and (3.3) with the heat loss term $\beta \theta$ has not considered by the earlier authors but we employ the "sensitized" form of reaction in Ayeni ${ }^{[7]}$, Okoya ${ }^{[10]}$ and Ajadi and Okoya ${ }^{[11]}$. We consider the special case of $r=-2$ corresponding to the" Sensitized" reaction for which $0 \leq \varepsilon<1$ in nonadiabatic system. Thus, equation (3.2) becomes

$\frac{d^{2} \theta}{d x^{2}}+\frac{\delta}{(1+\varepsilon \theta)^{2}} \exp \left(\frac{\theta}{1+\varepsilon \theta}\right)-\beta \theta=0$

Equation (3.8) that satisfies (3.3) is non-linear boundary value problem and not amendable to analytical methods of solution. Therefore, equation (3.8) together with (3.3) is solved numerically using shooting method techniques. 


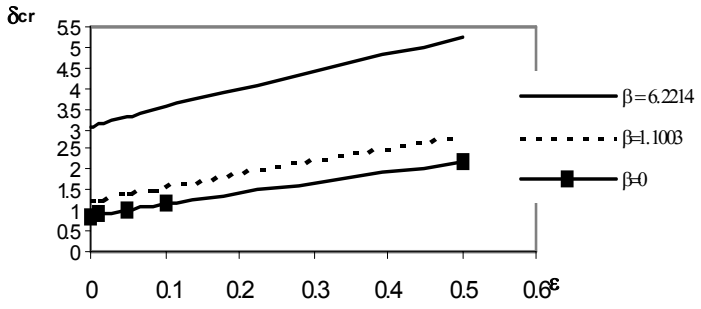

Fig. 1: Variation of critical Frank-Kamenestkii parameter $\delta_{\mathrm{cr}}$ with dimensionless activation energy $\varepsilon$ for various values of heat loss parameter $\beta$

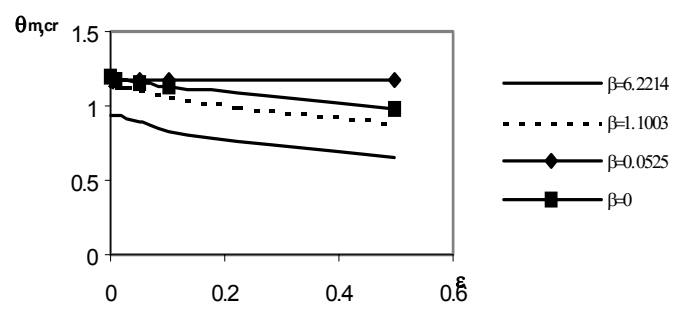

Fig. 2: Variation of critical ignition temperature $\theta_{\mathrm{m}, \mathrm{cr}}$ with dimensionless activation energy $\varepsilon$ for various values of heat loss parameter $\beta$

By these techniques, the boundary conditions in (3.3) becomes initial conditions

$\theta(-1)=0$ and $\theta^{\prime}(-1)=\sigma$

where $\sigma$ is unknown.

The numerical estimations of the critical values of Frank-Kamenetskii parameter $\delta_{\mathrm{cr}}$ and maximum ignition temperature $\theta_{\mathrm{m}, \mathrm{cr}}$ for various values of $\beta$ when $0 \leq \varepsilon<1$ are presented in the Table 1-4.

4. Numerical analysis and discussion: In this section, numerical solutions of thermal explosion of sensitized reaction with variable heat loss are presented. FrankKamenetskii parameter $\delta$, the activation energy parameter $\varepsilon$, the maximum temperature of the slab $\theta_{\mathrm{m}}$ and heat loss parameter $\beta$ characterize the reaction. Table 1-4 show typical variations of $\delta_{\mathrm{cr}}$ and $\theta_{\mathrm{m}, \mathrm{cr}}$ with $\varepsilon$ for various values of $\beta$. Notice from chemical and thermodynamics point of views when $\beta=0$, there is no heat exchange between the combustible material and the walls of the slab (i.e. adiabatic case), it is seen from Table 1 that $\delta_{\text {cr }}$ and $\theta_{\mathrm{m}, \mathrm{cr}}$ are monotonically increasing and decreasing respectively for increasing values of $\varepsilon$.

When $\beta>0$ (i.e. non-adiabatic case), Table 2-4 show that as heat loss parameter $\beta$ increases, critical ignition temperature $\theta_{\mathrm{m}, \mathrm{cr}}$ decreases and critical FrankKamenetskii parameter $\delta_{\text {cr }}$ increases for increasing values of $\varepsilon$.
In Fig. 1 and 2, the results in Table 1 to 4 are ascertained. As expected, the dimensionless critical ignition temperature and Frank-Kamenetskii parameter increases and decreases respectively as the heat loss parameter decrease monotonically. Values of $\delta$ and $\beta$ control explosion, when $\beta>\delta_{\text {cr }}$ in Table 4, the value of critical ignition temperature reduces which could prevent explosion from occurring.

\section{REFERENCES}

1. Semenov, N.N., 1959. Some Problems in Chemical Kinetics and Reactivity Part 1 and 2, Pergamon Press, London.

2. Dalton, F.S., 1966. Chain Reaction: An Introduction. Wiley, New York.

3. Frank-Kamenestkii, D.A., 1969. Diffusion and Heat Transfer in Chemical Kinetics. Plenum Press, New York.

4. Zeldovich, Ya.B., G.I. Barenblatt, V.B. Librovich and G.M. Makhviladze, 1985. The mathematical theory of combustion and explosions. Consultants Bureau, New York.

5. Kuo, K.K., 1986. Principle of Combustion. John Wiley and Sons.

6. Boddington, T., P, Gray and C. Robinson, 1979. Thermal explosions and the disappearance of criticality at small activation energies: Exact results for the slab. Proc. R. Soc. Lond., A , 368: 441-461.

7. Ayeni, R.O., 1982. On the explosion of Chainthermal reactions. J. Austral. Math. Soc. (Series B), 24: 194-202.

8. Ayeni, R.O., A.M. Okedoye, K.S. Adegbie and S.S. Okoya, 2004. Effect of heat loss on ignition times in the theory of branched chain explosions. J. Math. Assoc. Nigeria (Abacus), 31: 143-150.

9. Okoya, S.S., 2002. On the behaviour of solutions of a system of ordinary differential equations modelling branched chain reaction. Intl. Comm. Heat Mass Transfer, 28: 1169-1176.

10. Okoya, S.S., 2004. Reactive-diffusive equation with variable pre-exponential factor. Mech. Res. Comm. 31: 263-267.

11. Ajadi, S.O. and S.S. Okoya, 2004. The effect of variable pre-exponential factor on the Ignition time of a homogeneous system. Intl. Comm. Heat Mass Transfer, 31: 143-150. 